\title{
A Case Study of the 9 August 1988 South Atlantic Storm: Numerical Simulations of the Wave Activity
}

\author{
Valdir InNocentini* and ERnesto dos Santos Caetano Neto \\ Instituto de Pesquisas Meteorológicas, UNESP, Sao Paulo, Brazil
}

(Manuscript received 20 January 1995, in final form 4 October 1995)

\begin{abstract}
During 9-11 August 1988, a cyclone developed over Uruguay in the lee of the Andes Mountains and moved over the South Atlantic Ocean, where it redeveloped into an intense storm. This storm was responsible for unusual wave activity along the Brazilian shoreline from $22^{\circ}$ to $32^{\circ} \mathrm{S}$. The Brazilian news media reported the loss of at least one life, waves of $3 \mathrm{~m}$ and higher, and the disappearance of a drainage pipe, which weighed 8000 $\mathrm{kg}$, off the shores of Rio de Janeiro.

In this paper, the evolution of this intense storm and the associated ocean wave response is studied through European Centre for Medium-Range Weather Forecasts analyses, a hydrostatic limited-area meteorological model, and a second-generation prognostic wave model.

The atmospheric model results indicated the presence of a long-lived and large fetch with surface wind velocities higher than $12 \mathrm{~m} \mathrm{~s}^{-1}$ directed toward the coast. Some areas with velocities of $20 \mathrm{~m} \mathrm{~s}^{-1}$ were embedded in the fetch. The wave model forced by this wind field was able to simulate waves with a significant height of $8 \mathrm{~m}$ far from the coast and about $4 \mathrm{~m}$ in regions very close to the Brazilian coast in agreement with the occurrence reported at Rio de Janeiro. The swell propagation toward the coast of Rio de Janeiro was obstructed by a northeastward 10-m wind during the first 24-h period of the model's integration. During the second 24-h period, the fetch was still large and strong, but the obstacle was removed by a counterclockwise rotation of wind direction favoring the swell and windsea propagation toward the Rio de Janeiro coast.
\end{abstract}

\section{Introduction}

Many cyclones develop or intensify in the $30^{\circ}-50^{\circ}$ latitude belt in the region east of the Andes Cordillera. This cordillera has a nearly south-north direction, and although a large portion is located in the Tropics, its southern flank lies in a zone of westerly transient disturbances. Particularly in winter, when the disturbances tend to shift toward the north, the Andes Cordillera plays a significant role on the development or intensification of baroclinic synoptic-scale weather system. James and Anderson (1984) found a high incidence of baroclinic waves east of South America for the period July-August 1982. Gan and Rao (1991), studying 10 years (from January 1979 to December 1988) of synoptic surface charts for this region, identified two preferential regions for cyclogenesis located to the east of the Andes Cordillera - one over the Gulf of San Matias in Argentina $\left(42.5^{\circ} \mathrm{S}, 62.5^{\circ} \mathrm{W}\right)$ and the other over Uruguay (around $32.5^{\circ} \mathrm{S}, 55^{\circ} \mathrm{W}$ ). The former has higher

\footnotetext{
* Current affiliation: Instituto Nacional de Pesquisas Espaciais, SCT, São José dos Campos, Brazil.
}

Corresponding author address: Dr. Valdir Innocentini, Instituto Nacional de Pesquisas Espaciais, SCT, São José dos Campos 12201, Brazil. frequency in the summer season (December, January, and February ), and the latter has higher frequency during the winter season (June, July, and August). A recent study by Gan and Rao (1994) showed that the Andes Cordillera presence is associated with lee cyclogenesis for eastward-moving baroclinic disturbances.

An examination of satellite imagery reveals that many cyclones developing over Uruguay move to the South Atlantic Ocean, where they redevelop into intense storms. These storms can generate high waves, some of which can propagate toward the Brazilian coast.

It is well known to Brazilian fishermen that the period July-September usually offers adverse sailing conditions because of the formation of intense storms over the ocean. An example of a typical storm occurred in the period 9-11 August 1988. The storm was not felt over the continent but was responsible for unusual wave activity and flooding in some locations along the Brazilian shoreline from $22^{\circ}$ to $32^{\circ} \mathrm{S}$. On this occasion (and still now) no warning service was active, and precautionary measures were not taken by the public or those involved in marine activities within the coastal zone. The news media reported several instances of damages and the loss of at least one life. The Brazilian newspaper Jornal do Brasil from Rio de Janeiro wrote in its edition of 11 August 1988: "At 14 h yesterday . . . eight tubes of the drainage pipes at Leblon were 

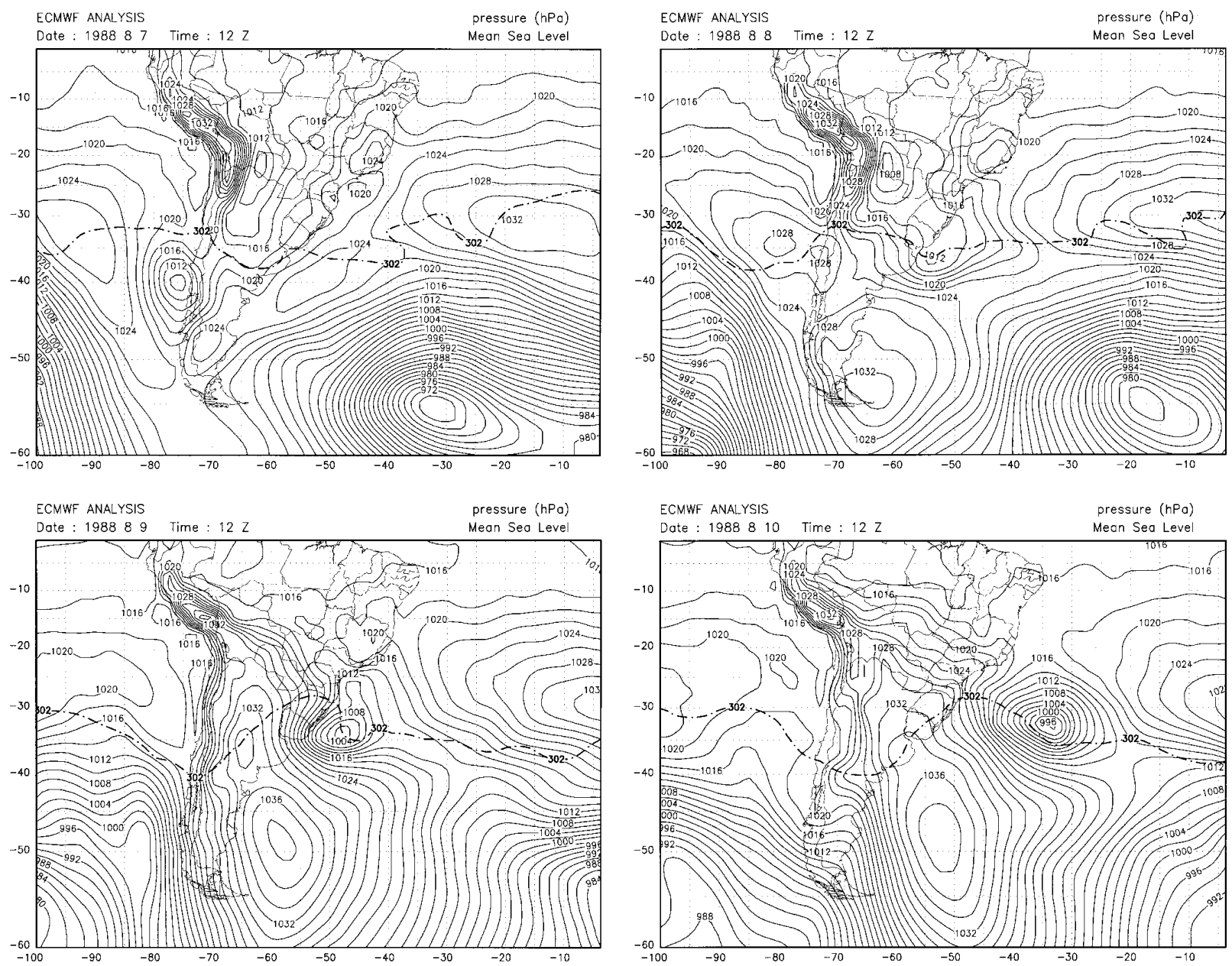

FIG. 1. ECMWF mean sea level pressure analyses (in hPa) and 700-hPa potential temperature of $302 \mathrm{~K}$ (point dashed) for 1200 UTC on (a) 7, (b) 8, (c) 9, and (d) 10 August 1988.

damaged by the water strength. One of them, with 8000 $\mathrm{kg}$, disappeared carried out by the sea . . . waves $3 \mathrm{~m}$ high caused several damages . . . people walking on the streets were forced to run inside the buildings trying to find protection in higher points.' In the 12,13 , and 14 August editions there are plenty of notices about deaths and damage.

The aim of this work is to hindcast, using numerical models, this elusive event. We employ a hydrostatic mesoscale meteorological model to simulate the storm and a second-generation wave model to hindcast the associated oceanic condition. The purpose is (i) to investigate the possibility of forecasting extreme ocean wave events due to lee cyclones developing over Uruguay and moving toward the ocean and (ii) to examine the surface wind evolution responsible for the intense wave activity observed in Rio de Janeiro. We begin by presenting a synoptic description of the case study in section 2 using European Centre for Medium-Range Weather Forecasts (ECMWF) analyses and comparing the storm's main features with the typical scenario of the Rocky Mountains lee cyclogenesis conceptualized by Bannon (1992). A brief description of the atmospheric and oceanic models is given in section 3 . The fields predicted by the atmospheric model are compared with ECMWF analyses in section 4, including a discussion of the results provided by both numerical models. The main conclusions are summarized in section 5.

\section{Synoptic overview}

A general overview of the synoptic characteristics is presented based on the ECMWF analyses. Figures 1 and 2 show the MSL (mean sea level) pressure and the 500-hPa heights for the period 1200 UTC 7 August 1988 through 1200 UTC 10 August, each 24 h. (The 500-hPa height on 10 August is omitted.) The fields are shown in a Mercator projection. Initially, (Fig. 1a) a surface low of $1010 \mathrm{hPa}$ at about $40^{\circ} \mathrm{S}, 75^{\circ} \mathrm{W}$ resides 

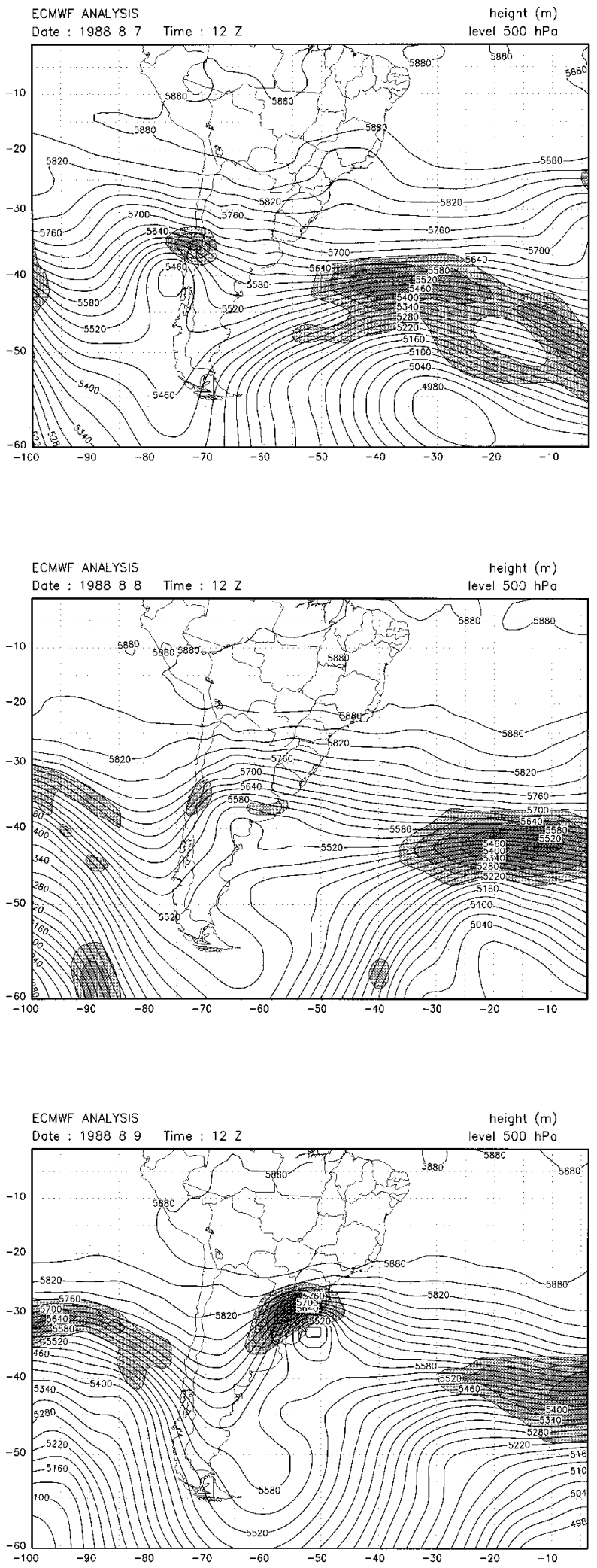

FIG. 2. ECMWF 500-hPa height (in $\mathrm{m}$ ) analyses and 500-hPa isotach higher than $30 \mathrm{~m} \mathrm{~s}^{-1}$ shaded at each $5 \mathrm{~m} \mathrm{~s}^{-1}$ interval for 1200 UTC on (a) 7, (b) 8, and (c) 9 August 1988. over the Pacific Ocean upstream the mountain. Over the continent, east of the Pacific low, a ridge is detected with a closed high of $1024 \mathrm{hPa}$ in the south (about $50^{\circ} \mathrm{S}, 70^{\circ} \mathrm{W}$ ). At $500 \mathrm{hPa}$ (Fig. 2a) the cyclone is nearly over the surface low $\left(40^{\circ} \mathrm{S}, 75^{\circ} \mathrm{W}\right)$, exhibiting a barotropic structure. The surface high at about $50^{\circ} \mathrm{S}$, $70^{\circ} \mathrm{W}$ is supported by a high-level ridge. The next 24$\mathrm{h}$ analysis on 8 August shows the low over the Pacific Ocean being replaced by a high center (Fig. 1b). A lee cyclone is visible at low levels (nearly $37^{\circ} \mathrm{S}, 54^{\circ} \mathrm{W}$ ) northeast downwind from the previous low location and with a slightly higher pressure. At higher levels the low center (nearly $40^{\circ} \mathrm{S}, 63^{\circ} \mathrm{W}$ in Fig. $2 \mathrm{~b}$ ) is displaced eastward, conserving its previous latitude. The surface high center was filled by $8 \mathrm{hPa}$ as it moved southeast (about $55^{\circ} \mathrm{S}, 65^{\circ} \mathrm{W}$ in Fig. 1b). By 9 August the surface low pressure over the Atlantic Ocean deepened $8 \mathrm{hPa}$, and its accompanying $500-\mathrm{hPa}$ low center was very well defined (about $35^{\circ} \mathrm{S}, 50^{\circ} \mathrm{W}$ in Figs. 1c and 2c). At this time, the upper-level and surface low pressure centers are displayed in a situation favorable to the intensification of the synoptic system; the cold (warm) air mass flow below (above) the 500-hPa (surface) low pressure center is a classic baroclinic situation in which the synoptic system will deepen in the forthcoming hours. This is confirmed by the $302 \mathrm{~K}$ potential temperature configuration at the $700-\mathrm{hPa}$ level (Fig. 1c). The 500-hPa jet streak pictured in Fig. 2c reveals the low-level cyclone developing below the exit region, where the semigeostrophic theory predicts an indirect cross-stream circulation favorable to decreasing the surface pressure due to cyclonic advection aloft. This ratifies the baroclinic character of the event (Sanders 1986).

Although it is not relevant to the main purpose of this paper, at this point it is instructive to compare the situation described above with the typical scenario of lee cyclogenesis occurring east of the Rocky Mountains of North America, as described by Bannon (1992), since both topographies have north-south configuration. While the cyclone over the Pacific Ocean is evident in both cases during the early stage of lee cyclogenesis, the anticyclone reported here is located poleward, not equatorward as in the case of the Rocky Mountains. In this stage the Andes cyclone has a barotropic structure, in contrast with the baroclinic structure of the Rocky Mountains. No trough is observed on the lee side of the Rocky Mountains in advance of the moving cyclone, as pointed out by Bannon. In fact, an inverted ridge is visible by 7 August on the lee side followed by a trough nearly $40^{\circ} \mathrm{S}, 62^{\circ} \mathrm{W}$. The lee cyclone develops equatorward of the Pacific Ocean cyclone location in both cases, but no preceding anticyclone first moving equatorward and then poleward in advance of the lee cyclone is noticed, as in the scenario pictured by Bannon. Instead, the anticyclone initially in advance of the Pacific Ocean low moved more slowly, presenting an impressive increase in its central pressure. 
On 10 August the surface low maintained its deepening rate of $8 \mathrm{hPa}$ day $^{-1}$ from the previous $24 \mathrm{~h}$ and accelerated its eastward motion (Fig. 1d). By $11 \mathrm{Au}-$ gust the deepening rate was reduced, and the partner anticyclone exhibited a decrease in its central pressure value (not shown).

Wash et al. (1992) classified a cyclone as explosive when the central pressure drops at least $1 \mathrm{hPa}$ per hour for a time period of at least $12 \mathrm{~h}$. In this sense, the case reported here, as revealed by the global analyses, can be considered a very weak case. However, the criteria adopted by Wash et al. (1992) were based on investigations of cases occurring in the eastern coastal region of North America, recognized as one of the most active cyclogenetic regions of the earth. To our knowledge there is no similar study applied to South American developing cyclones. Also, the analysis performed by ECMWF is based on a blending between the first guess (a 6-h period simulation) and observations; once the cyclone described here develops in a very poor data region - and even the best operational numerical models usually fail in reproducing observed deepening rates of central pressures (Kuo and Reed 1988) - one can not safely classify this cyclone as nonexplosive.

\section{Models description}

In this work an LAM (limited-area atmospheric model) and a SWM (ocean surface wave model forced by wind) are used. The coupling between the two models is performed in a one-way form. The LAM simulates the meteorological episode and provides a 10-m wind field at 3-h intervals to force the SWM.

The use of wind generated by a limited-area atmospheric model is more suitable to study the ocean wave evolution, because many atmospheric mesoscale systems can be explicitly simulated, and generally more realistic surface winds are obtained than in global models. Also, the analyses and forecasts provided by international centers operating global models are available only at each 6- or 12-h interval, reducing the frequency to update the wind forcing for ocean waves.

Only a brief description of the numerical models is given here. More detailed description can been found in Nagata et al. (1986) and Innocentini and Caetano Neto (1994) regarding LAM and SWM, respectively.

\section{a. LAM}

The LAM is a flux-form primitive equation model developed by Yamagishi (1980) and Tatsumi (1983), researchers of the Numerical Prediction Division of the Japan Meteorological Agency, and modified by Nagata and Ogura (1991). The version used here has 14 layers vertically in the sigma coordinate system $[\sigma \equiv(p$ $\left.\left.-p_{\text {top }}\right) /\left(p_{\text {surface }}-p_{\text {top }}\right)\right]$ with $p_{\text {top }}=100 \mathrm{hPa}$. The layers are defined by the $15 \sigma$ levels corresponding to the pressures 1000, 990, 970, 940, 900, 850, 790, 720, 640,
$550,450,350,250,150$, and $100 \mathrm{hPa}$ for $p_{\text {surface }}=1000$ $\mathrm{hPa}$. The prognostic variables $\Pi \equiv p_{\text {surface }}-p_{\text {top }}, u, v$, $\theta$, and specific humidity $q$ are placed in the middle of each layer, and the diagnostic variable $\omega \equiv d p / d t$ in the levels. As usually assumed in numerical models with this kind of vertical coordinate, the surface pressure tendency equation is formulated so that $\omega=0$ at the surface and the top. The geopotential height is calculated in the middle of each layer by the vertical integration of the hydrostatic equation.

The boundary-layer processes are evaluated using the level 2 closure model (Mellor and Yamada 1974), and the surface fluxes are calculated by the similarity theory with the universal functions defined in Businger et al. (1971). The sea surface temperature, fixed during the integration, is obtained from monthly climatic data. The precipitation processes considered are the gridscale condensation and cumulus convection parameterized using the Kuo scheme (Kuo 1965) with modifications suggested by Geleyn (1985). The evaporative effect of grid-scale precipitation is incorporated explicitly, as described by Nagata and Ogura (1991). The prognostic variables are computed by the two-time-step scheme proposed by Tatsumi (1983). The horizontal grid spacing is constant on the projected map, which can be the polar-stereographic or the cylindrical of Mercator.

The horizontal domain used by the atmospheric model to simulate the case study is represented in Fig. 4. It consists of 73 and 55 grid points in the east and north directions, respectively, in a Mercator projection. The grid distance on this map is $104.125 \mathrm{~km}$ true at $30^{\circ}$ latitude. This resolution is appropriate to capture the broad-scale synoptic features of the event but not to simulate explicitly the mesoscale embedded on it. The horizontal resolution of the atmospheric model is fundamental in wave forecasting. For the Gorbush Storm, in the Mediterranean Sea Dell'Osso et al. (1992) simulated the wave activity with two ECMWF numerical models - one the global model with resolution T106 and the other the limited-area model with resolution T333, corresponding approximately to 125 and $40 \mathrm{~km}$, respectively. Dell'Osso et al. (1992) obtained realistic wave height forecasting only with the $10-\mathrm{m}$ winds provided by the limited-area model.

The ECMWF global model resolution in 1988 was T106, very similar to the resolution used in the present research. However, as it will be shown, the 10-m winds obtained with the LAM model are stronger than those provided by ECMWF analysis, and the ocean waves generated are due to a long-lived and very large fetch originated from the synoptic scale, and therefore, the resolution used here seems to be satisfactory for the present purpose.

The model is integrated for $48 \mathrm{~h}$ initialized with the ECMWF global analysis for 1200 UTC 9 August 1988, when the lee cyclone is well defined (Fig. 1c). The analyses used are available at 1000-, 850-, 700-, 500-, 
$300-, 200-$, and $100-\mathrm{hPa}$ pressure levels with $2.5^{\circ}$ of horizontal resolution, and the variables are interpolated to the $\sigma$ levels and model grid points using a cubic spline.

At each time step the model prognostic variables are modified at the outermost five grid points along the lateral boundary. The objective is to include the tendency of the global analysis in forthcoming time steps. This is performed with the procedure proposed by Davies (1976). The analyses for the simulation reported here are available at only each $24 \mathrm{~h}$; they are obtained at each time step by linear temporal interpolation of two subsequent analyses.

\section{b. $S W M$}

The SWM is a second-generation wave model incorporating advection, refraction, shoaling, dissipation due to the bottom, input of energy due to the horizontal wind $10 \mathrm{~m}$ above the surface, dissipation due to the wave breaking, and conservative nonlinear interactions. It is based on the energy balance equation written for the wave spectral variance. It follows the model developed by Golding (1983) in many aspects. Concerning the parameterization of physical processes, the main distinctions between the two models are the following.

- The advective process is performed using a semiLagrangian scheme (Bates and Mcdonald 1982).

- The sources terms (generation and dissipation due to the wave-breaking) and nonlinear interactions are tuned to fit the empirical Sanders's duration-limited growth curve (Sanders et al. 1981).

- The nonlinear interactions are performed so that the windsea is reshaped to resemble the Kruseman spectrum (Janssen et al. 1984).

The wave spectrum is represented at each grid point in 13 frequencies corresponding to the periods $1,2,3$, $4,5,6,7,8,10,13,16,20$, and $25 \mathrm{~s}$, and 36 directions with an angular interval of $10^{\circ}$.

The only physical process requiring boundary conditions is the advection. At the coast the spectral energy is imposed equal to zero. At the open ocean boundaries, since the semi-Lagrangian scheme requires the value of the advected variable in the point where "the parcel" was located in the previous time step, when this point is outside the domain (energy entering into the domain) the nearest boundary point value is used. If this point is inside the domain, the semi-Lagrangian scheme is applied normally.

The empirical Sanders's duration-limited growth curve relates the 10-m wind speed with the significant wave height. Once this curve is used to tune the wave model, the LAM must provide this wind field to force the wave. It is obtained by the relation

$$
|u(z)|=\frac{u^{*}}{0.41} \ln \left(\frac{z}{z_{0}}\right),
$$

where $z_{0}=0.05 \mathrm{~m}$ and $u^{*}$ is the friction velocity. First, $u^{*}$ is calculated using $|u|$ at the first LAM level, imposing $|u|=0$ at $z=z_{0}$. With $u *$ computed, the same relation is evoked again to compute using $|u|$ at $z$ $=10 \mathrm{~m}$. The wind direction at the first model level above $10 \mathrm{~m}$ is assumed at $z=10 \mathrm{~m}$.

The SWM is integrated in the same domain and grid mesh as the LAM (see Fig. 4 in section 4). The initial wave field is obtained running the SWM for $9 \mathrm{~h}$ from an ocean state at rest. The forcing is the initial surface wind. The water depth is assumed constant and equal to $1000 \mathrm{~m}$, which means that refraction, shoaling, and bottom dissipation are neglected. This is a reasonable assumption, because the ocean depth in the region considered relevant for this study is smaller than $200 \mathrm{~m}$ only about $200 \mathrm{~km}$ from the coast. The $10-\mathrm{m}$ wind data are used to update the wind forcing at each 3-h period during the SWM integration.

An intercomparison among several operational models for idealized experiments described in The SWAMP Group (1985) are presented in Innocentini and Caetano Neto (1994). This study shows that the wave model employed in this research is satisfactory as a secondgeneration wave model. However, the wave model is not considered in its final version. A formulation controlling how fast the windsea average direction aligns with the wind direction is currently being investigated. The results are being compared with the method adopted in the VAG second-generation model (Guillaume 1990) and with the EXACT-NL third-generation model (Hasselmann and Hasselmann 1985). This procedure will reduce the shortcoming detected in secondgeneration wave models submitted to situations of varying wind directions and wind velocities (Günther et al. 1981; van Vledder and Holthuijsen 1993).

Recently, Innocentini (1995) studied the wave activity simulated by SWM in the Mediterranean Sea forced by the Gorbush Storm (Dell'Osso et al. 1992). Figure 3 depicts the significant wave height, mean direction, and mean period at Malta for the SWM (continuous line) and the third-generation wave model employed by them. (The dotted line refers to the simulation with forcing given by the atmospheric model T106, and the broken-dotted line refers to the simulation with forcing given by the atmospheric model T333.) The SWM results were obtained with the $10-\mathrm{m}$ wind provided by the ECMWF limited area with resolution T333. There is a general tendency for SWM to present higher waves in the maximums and smaller waves in the minimums, but the difference always is less than $0.5 \mathrm{~m}$. Greater discrepancies are expected in mean period and mean direction, since the third-generation wave model uses 25 frequencies and has longer response time in aligning the directional spectrum with wind (The SWAMP Group 1985). However, as revealed by Figs. 3b,c, the difference in mean period and mean direction never exceeds $1.5 \mathrm{~s}$ and $30^{\circ}$ during the 


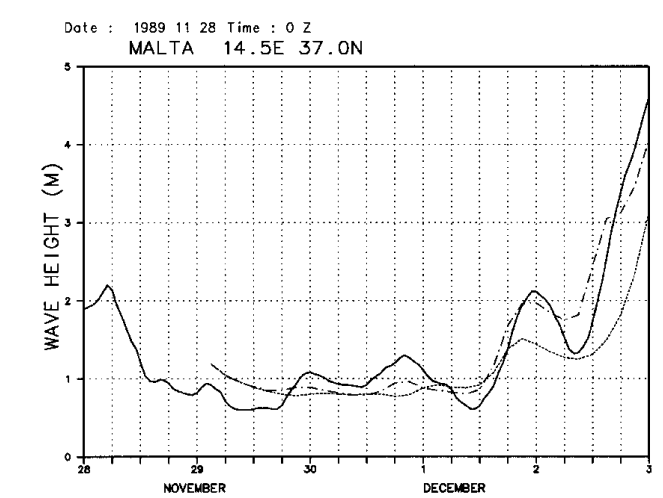

Gros: cow/ars
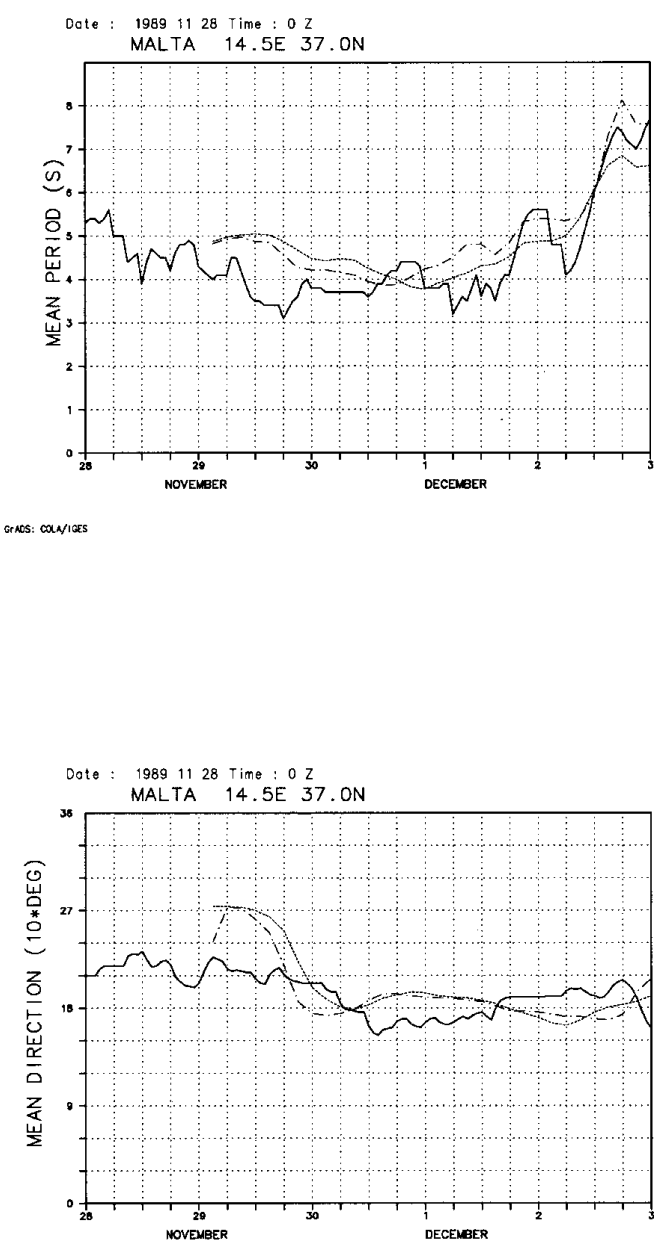

FIG. 3. Evolution of (a) significant wave height in meters, (b) mean period in seconds, and (c) mean direction in degrees for the Gorbush Storm at Malta. The SWM simulation (continuos line) is obtained with the wind from the LAM, while the third-generation wave simulations are obtained with wind from limited-area (broken dotted) and global models (dotted). most active period of the storm (from 2 to 3 December 1989).

\section{Simulation of the 9 August 1988 storm}

The ECMWF global analysis for $T=1200$ UTC 9 August 1988 is used to initialize the LAM, with 1200 UTC 10 August 1988 and 1200 UTC 11 August 1988 analyses updating the boundary values. The LAM supplies the 10-m wind data at each 3-h period to force the wave generation in SWM. The numerical procedures adopted were described in section 3 .

The 10-m wind obtained with LAM forecasting at $T$ $+0 \mathrm{~h}, T+24 \mathrm{~h}$, and $T+48 \mathrm{~h}$ are depicted in Fig. 4 . Initially the maximum velocity center of $12 \mathrm{~m} \mathrm{~s}^{-1}$ is located around $38^{\circ} \mathrm{S}, 45^{\circ} \mathrm{W}$ (Fig. 4a). The next forecasts show this maximum enhancing to $20 \mathrm{~m} \mathrm{~s}^{-1}$ and moving northeastward in the first $24 \mathrm{~h}$ and southeastward in the next period (Figs. 4b,c). The most remarkable feature of these fields is the large region around the point $35^{\circ} \mathrm{S}, 40^{\circ} \mathrm{W}$ at $T+24 \mathrm{~h}$ (Fig. $4 \mathrm{~b}$ ) with velocity higher than $12 \mathrm{~m} \mathrm{~s}^{-1}$ directed toward the coast, except in its north flank and near the coast. At $T+48$ h (Fig. 4c) the area around $25^{\circ} \mathrm{S}, 42^{\circ} \mathrm{W}$ (near the coast of Rio de Janeiro) embedded in this large region experiences a counterclockwise rotation of wind direction decreasing its eastward component in relation to $T$ $+24 \mathrm{~h}$. The remaining part of this large region displays nearly no change in wind direction. Then, one can expect a vigorous windsea built at the area, with wind speed greater than $20 \mathrm{~m} \mathrm{~s}^{-1}$ being propagated toward the coast. The large fetch and counterclockwise wind rotation observed above favor the swell propagation along the shoreline $22^{\circ}-26^{\circ} \mathrm{S}$, where Rio de Janeiro is located.

Once the 10-m wind is obtained in the LAM through a crude interpolation (see section 3), a comparison with this field given by a global analysis is instructive. Figure 5 shows the 10-m wind ECMWF analyses at 1200 UTC 9 and 10 August 1988. Comparing Figs. 4a and 5a at 9 August 1988 one can note that the two maximum centers of $16 \mathrm{~m} \mathrm{~s}^{-1}$ north and south of the point $35^{\circ} \mathrm{S}, 50^{\circ} \mathrm{W}$ presented by the global analysis are not captured in the LAM initial field. Some smoothing should be expected, because the fields used initially to feed the LAM are obtained with a very coarse vertical resolution near the surface. However, the maximum location and the direction agree reasonably. The next 24h ECMWF analysis (Fig. 5b) shows a minimum of 8 $\mathrm{m} \mathrm{s}^{-1}$ at $34^{\circ} \mathrm{S}, 34^{\circ} \mathrm{W}$ (the position of the cyclone center) surrounded by two maximums of 16 and $18 \mathrm{~m} \mathrm{~s}^{-1}$. At this time, this minimum simulated by the LAM is nearly in the same position (Fig. 4b) but with a value of $4 \mathrm{~m} \mathrm{~s}^{-1}$ and elongated in the northwest direction. The LAM is able to simulate a maximum of 20 $\mathrm{m} \mathrm{s}^{-1}-2 \mathrm{~m} \mathrm{~s}^{-1}$ greater than that provided by the ECMWF analysis. However, the closed contour of 16 $\mathrm{m} \mathrm{s}^{-1}$ around the point $28^{\circ} \mathrm{S}, 32^{\circ} \mathrm{W}$ reported by the 

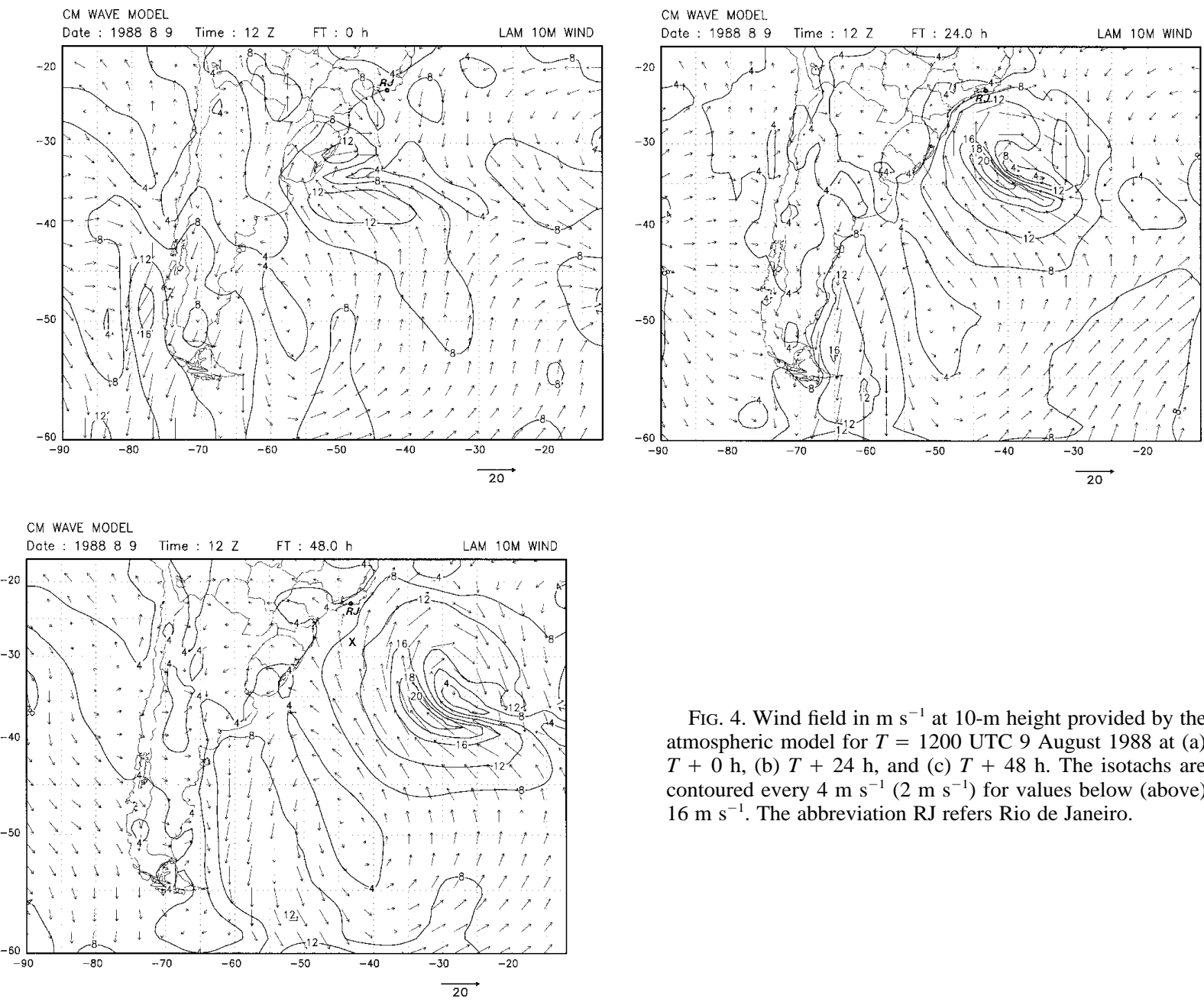

FIG. 4. Wind field in $\mathrm{m} \mathrm{s}^{-1}$ at $10-\mathrm{m}$ height provided by the atmospheric model for $T=1200$ UTC 9 August 1988 at (a) $T+0 \mathrm{~h}$, (b) $T+24 \mathrm{~h}$, and (c) $T+48 \mathrm{~h}$. The isotachs are contoured every $4 \mathrm{~m} \mathrm{~s}^{-1}\left(2 \mathrm{~m} \mathrm{~s}^{-1}\right)$ for values below (above) $16 \mathrm{~m} \mathrm{~s}^{-1}$. The abbreviation RJ refers Rio de Janeiro.

analysis is not reproduced by the LAM. At 1200 UTC 11 August 1988 the discrepancies noted above are still present (the ECMWF analysis for this time is not shown): (i) the minimum is smaller in the LAM; (ii) the maximum southwest to the minimum is stronger in the LAM; and (iii) the maximum north of the minimum is not captured by the LAM. Although these differences must be investigated in future research, we conclude that the main feature of the fetch responsible for the intense wave activity propagating toward the south Brazilian coast is reproduced by the LAM and satisfies the main purpose of this paper.

Figure 6 presents the surface pressure and the isotherm $\theta=302 \mathrm{~K}$ at the 700 -hPa level provided by the LAM for $T=1200$ UTC 9 August 1988 at forecasting time (a) $T+24 \mathrm{~h}$ and (b) $T+48 \mathrm{~h}$. The surface low falls about $12 \mathrm{hPa}$ in the first $24 \mathrm{~h}$ and $6 \mathrm{hPa}$ in the next 24-h period during its eastward displacement. In the first 24-h period (Fig. 6a), a comparison with ECMWF analyses (Fig. 1d) reveals a stronger deepening rate and a slower eastward motion for the low center sim- ulated by the LAM. The anticyclone southwest of the low center presents nearly the same location in the ECMWF analyses, but the filling rate is smaller in the LAM. The horizontal pressure gradient simulated by the LAM is stronger than that presented by the analysis, justifying the stronger LAM 10-m wind speed. The second 24-h period (Fig. 6b) maintains the main features noted above. Although the low center pressure deepening rate does not suggest an explosive cyclogenesis as established by Wash et al. (1992), a close examination in LAM results at each $3 \mathrm{~h}$ shows a pressure fall of $10 \mathrm{hPa}$ between $T+6 \mathrm{~h}$ and $T+18 \mathrm{~h}$ (not shown). In particular, the LAM exhibits a decrease of $4 \mathrm{hPa}$ between $T+15 \mathrm{~h}$ and $T+18 \mathrm{~h}$.

Figure 7 shows the significant wave height $H_{s}$ and average wave direction $D_{\text {ave }}$ at $T+0 \mathrm{~h}, T+24 \mathrm{~h}$, and $T+48 \mathrm{~h}$. At $T+0 \mathrm{~h}$ the sea state is obtained by the 10-m wind at 1200 UTC 9 August 1988 frozen for a time period of $9 \mathrm{~h}$. The initial fields $H_{s}$ and $D_{\text {ave }}$ closely resemble the location of wind maximum and wind direction but not exactly because the initial spinup of 

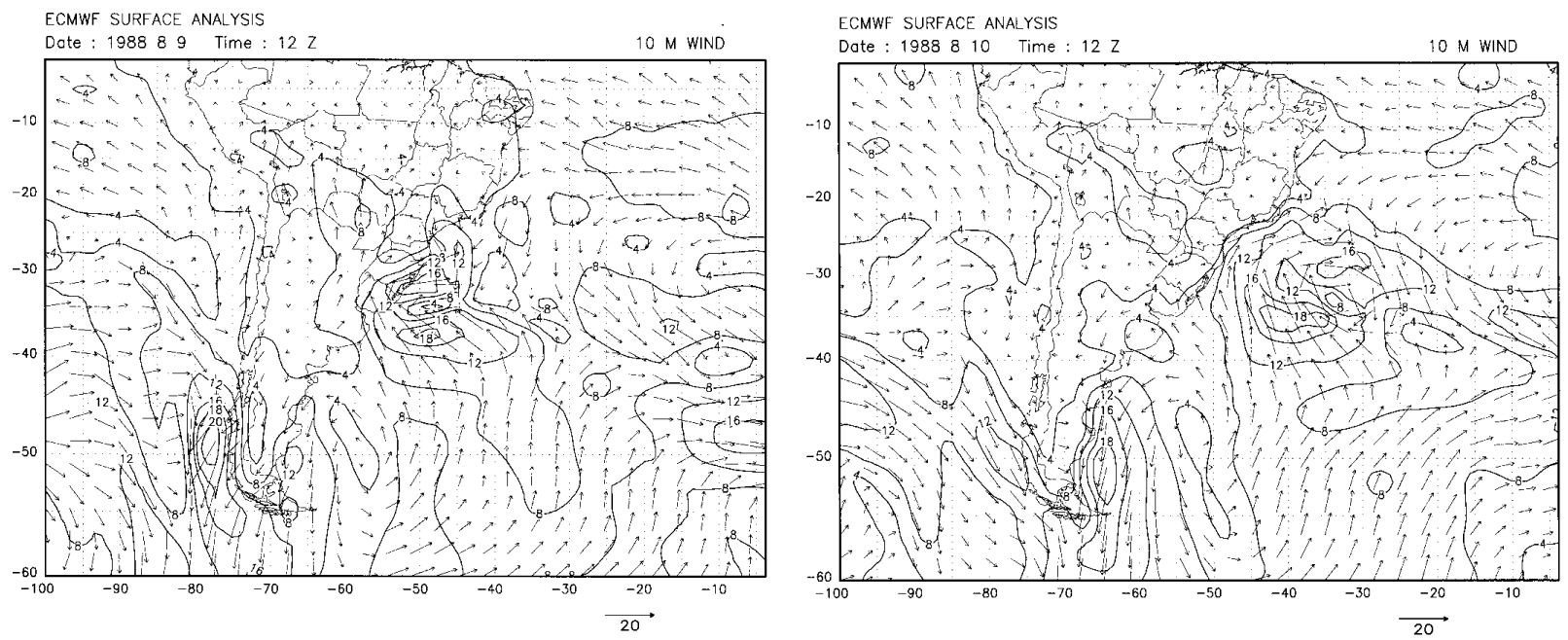

FIG. 5. ECMWF 10-m wind analyses for 1200 UTC on (a) 9 and (b) 10 August 1988. The isotachs are contoured every $4 \mathrm{~m} \mathrm{~s}^{-1}\left(2 \mathrm{~m} \mathrm{~s}^{-1}\right)$ for values below (above) $16 \mathrm{~m} \mathrm{~s}^{-1}$.

SWM is obtained with the advection due to the wave group velocity switched on. The time period of $9 \mathrm{~h}$ to spin up wave models is an arbitrary practice usually adopted (Golding 1983) before the availability of altimeter wave height data obtained by satellite. Currently several operational wave models are initialized with the assimilation of satellite data (Breivik and Reistad 1994). The wave height of $3 \mathrm{~m}$ built in the region with $10-\mathrm{m}$ wind of $12 \mathrm{~m} \mathrm{~s}^{-1}$ (Fig. 7a) does not correspond to a fully developed spectrum, which requires this wind value to blow for about $15 \mathrm{~h}$ without any variation (Innocentini and Caetano Neto 1994). However, there is no reason to force the wave models starting with a fully developed spectrum, because the 10-m wind is a consequence of the cyclone developing and moving from the continent toward the coast. An in- spection in the 10-m wind analysis $12 \mathrm{~h}$ earlier (not shown) reveals that the maximum wind was $12 \mathrm{~m} \mathrm{~s}^{-1}$ concentrated in a smaller area around $45^{\circ} \mathrm{S}, 52^{\circ} \mathrm{W}$ directed toward the coast. Since at the initial time the wind is weaker than that simulated in the forthcoming hours and the fetch is still not well characterized, one can guess that the time period chosen to spin the wave model does not significantly affect the main features of the wave simulation. Anyway, an experiment was carried out with the initial $10-\mathrm{m}$ wind field frozen for a time period of $48 \mathrm{~h}$. The results obtained (not shown) were very similar.

At $T+24 \mathrm{~h}$, high waves are evident in many parts of the coast in south of Brazil; $H_{s} \approx 4-5 \mathrm{~m}$ are found at $30^{\circ} \mathrm{S}, 50^{\circ} \mathrm{W}$ (Fig. $7 \mathrm{~b}$ ). Qualitatively, stronger winds generate waves with smaller frequencies, which have
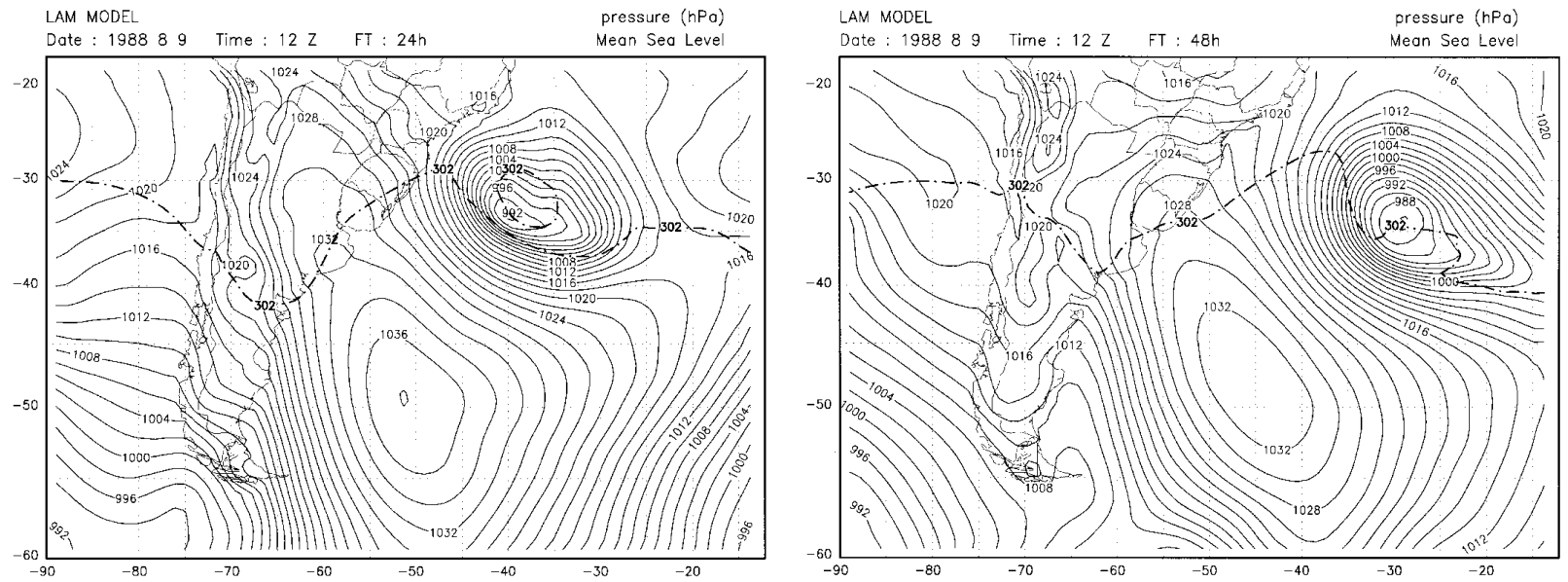

FIG. 6. Surface pressure (in $\mathrm{hPa}$ ) and $700-\mathrm{hPa}$ potential temperature of $302 \mathrm{~K}$ provided by the atmospheric model for $T=1200$ UTC 9 August 1988 at forecast time (a) $T+24 \mathrm{~h}$ and (b) $T+48 \mathrm{~h}$. 

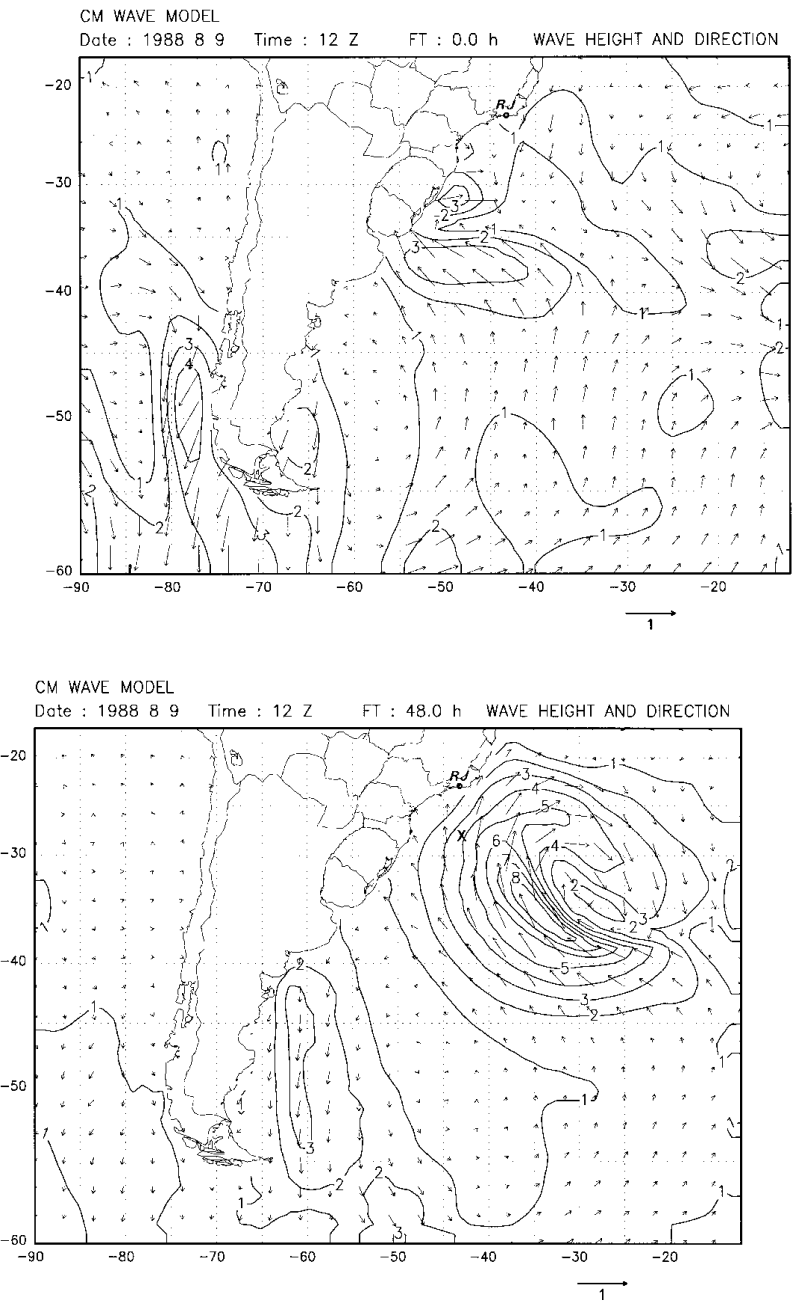

greater group velocities and are propagated faster. When the waves reach a region with smaller wind speed than in the source region, part of this energy (that in the spectral band with higher frequencies) becomes windsea suffering an angular relaxation toward the wind direction, and the spectrum is reshaped. The remainder of the energy stays in lower frequencies with its original group velocity and direction. This situation seems to take place around Rio de Janeiro (nearly $23^{\circ} \mathrm{S}$ ), as depicted in Fig. $7 \mathrm{~b}$, where the wind blows northeastward. Conversely, around the point $30^{\circ} \mathrm{S}$, $48^{\circ} \mathrm{W}$ the wind direction is almost the same of the incoming wave energy.

From $T+24 \mathrm{~h}$ to $T+48 \mathrm{~h}$ (Fig. 7c) the wind changes its direction around Rio de Janeiro, allowing the swell and windsea propagation toward the coast. The $H_{s}>3 \mathrm{~m}$ contours extend northward, reaching regions very close to the coast of Rio de Janeiro with direction toward the coast. Figure 8 presents the spectrum at $28^{\circ} \mathrm{S}, 43^{\circ} \mathrm{W}$ (indicated by $\mathrm{X}$ in Fig. $4 \mathrm{c}$ ) for $T$ $+45 \mathrm{~h}$. One can note the swell building along the north

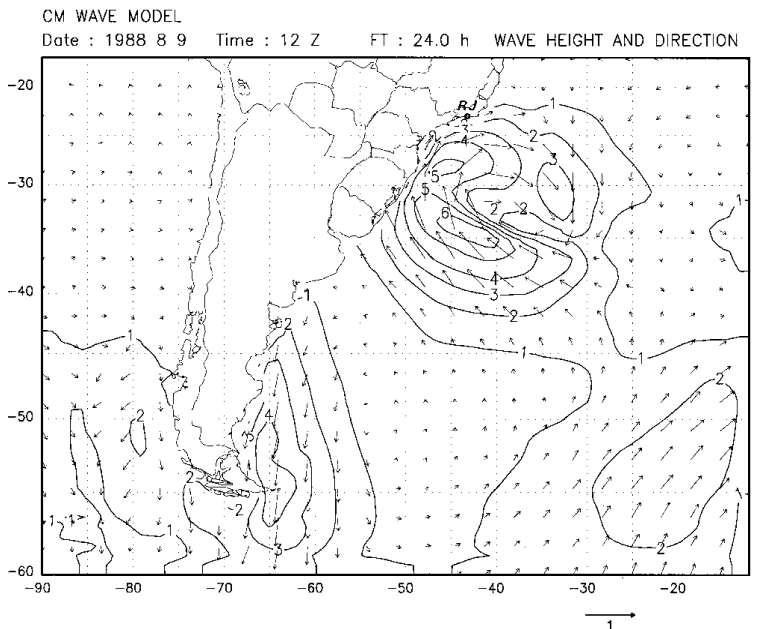

FIG. 7. Isolines of significant wave height $(\mathrm{m})$ and average direction provided by the wave model for $T=1200$ UTC 9 August 1988 at forecasting time (a) $T+0 \mathrm{~h}$, (b) $T+24 \mathrm{~h}$, and (c) $T+48 \mathrm{~h}$. Vectors are plotted at each three grid points.

direction (nearly the local wind direction) and energy in smaller frequencies in the northwest quadrant corresponding to the swell that has propagated from the region with stronger wind southeast of this point.

The windsea generation in a location where waves are being propagated from a remote region with higher wind speed depicted above is a physical constraint that must be captured by wave models. However, this effect is too rapidly reproduced by many second-generation wave models (as the one employed here), in contrast with third-generation models, where the nonlinear interactions explicitly build the windsea spectrum (The SWAMP Group 1985). Probably a third-generation wave model could show greater details in the wave field along the coast of Rio de Janeiro even before $T+24 \mathrm{~h}$.

\section{Summary and conclusions}

During the Southern Hemisphere winter a high frequency of cyclogenesis occurs over Uruguay generated 


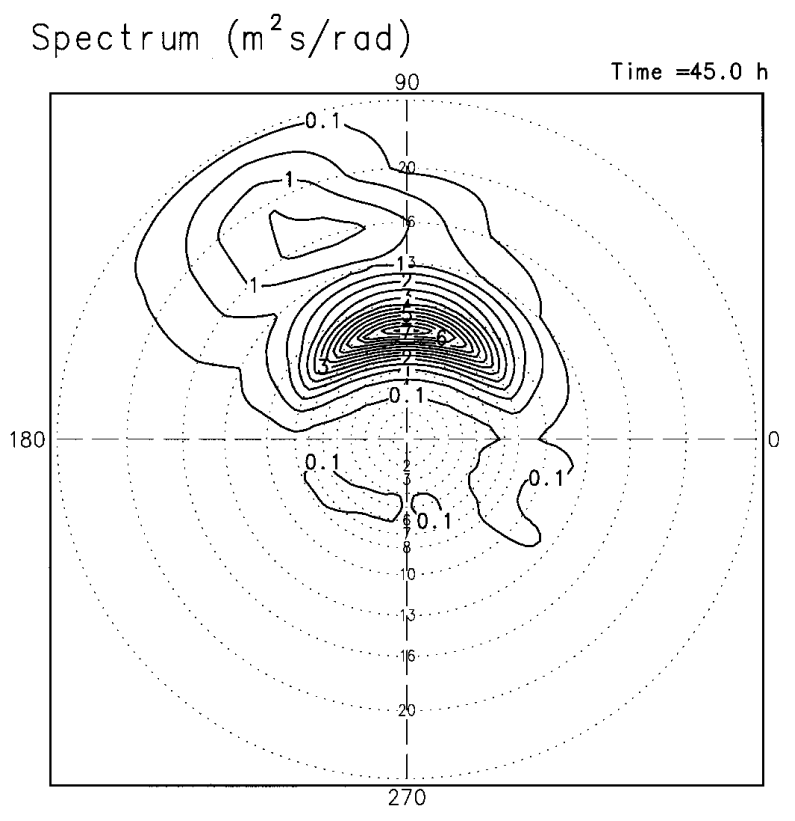

FIG. 8. Two-dimensional spectrum at $28^{\circ} \mathrm{S}, 43^{\circ} \mathrm{W}$, indicated by $\mathrm{X}$ in Fig. 4c, for $T+45 \mathrm{~h}$. The circles refer to the periods 1, 2, 4, 5, 6, $7,8,10,13,16,10$, and $25 \mathrm{~s}$. The directions are defined counterclockwise from eastward, which corresponds to 0 . The contour interval chosen is $0.5 \mathrm{~m}^{2} \mathrm{~s}(\mathrm{rad})^{-1}$.

by the effect of the Andes Cordillera on westerly atmospheric baroclinic waves. Many cases of intense ocean wave activity during this period are observed, probably due to cyclones of this kind moving toward the South Atlantic and evolving into intense storms. The 9 August 1988 storm was a typical example. The ocean waves reached the South Brazilian coast, causing severe instances of damage and the loss of at least one life, as reported by the Brazilian news media. Waves 3 $\mathrm{m}$ high observed at Rio de Janeiro and the disappearance of a 8000-kg-weight tube from a drainage pipe illustrated the strength of the waves.

This elusive phenomenon is studied in this paper through ECMWF analyses, a hydrostatic LAM, and an SWM.

The ECMWF analyses of MSL pressure and 500$\mathrm{hPa}$ heights show many features similar to the typical scenario of lee cyclogenesis depicted by Bannon (1992). Also, some discrepancies are noted, as, for example, the lack of a trough in the lee side of the Andes Cordillera in advance of the Pacific Ocean low center, and the lack of a preceding anticyclone first moving equatorward and then poleward.

After the lee cyclone has been formed over the Atlantic Ocean at $T=1200$ UTC 9 August 1988, the ECMWF analyses present a deepening rate of $8 \mathrm{hPa}$ day $^{-1}$, which does not configure an explosive cyclogenesis in the sense suggested by Wash et al. (1992). The LAM atmospheric model simulates a deepening rate of $10 \mathrm{hPa}$ in $12 \mathrm{~h}$, and in some instances, $4 \mathrm{hPa}$ per $3 \mathrm{~h}$ (not shown). However, even the best operational numerical model fails in reproducing observed deepening rates in explosive cyclones (Kuo and Reed 1988).

The ECMWF 10-m wind agrees with this field simulated by the atmospheric model concerning the location of the maximum and minimum intensities. The discrepancies noted are that (i) the minimum wind in the cyclone center is smaller in the LAM, (ii) the maximum wind southwest of the cyclone center is stronger in the LAM, and (iii) a maximum wind north of the cyclone center shown by the analyses is not captured in the LAM simulation. Despite these differences and the coarse mesh grid employed by the LAM, the longlived and large-fetch-generating waves propagating toward the south Brazilian coast are reproduced.

The SWM simulates waves with significant height $H_{s} \approx 4-5 \mathrm{~m}$ south of Brazil at $T+24 \mathrm{~h}$. At this time a northeastward 10-m wind around Rio de Janeiro seems to obstruct the swell propagation from the fetch. However, as the surface cyclone moves eastward from $T+24 \mathrm{~h}$ through $T+48 \mathrm{~h}$ this obstruction is removed and high waves directed to the coast achieve regions close to Rio de Janeiro. Then the two-dimensional spectrum around this region presents two peaks in distinct directions and frequencies, revealing the simultaneous presence of swell and windsea.

Although the objectives of this research, concerning the elucidation of the cause related to the intense wave activity observed in Rio de Janeiro on 10 August 1988 and the ability in its forecasting, were successfully achieved, further research must be done on the effect of more realistic nonlinear interactions, as used in thirdgeneration wave models. Also, how dynamics and thermodynamics interact to help the cyclogenesis over Uruguay and its displacement over the ocean must be investigated.

Acknowledgments. This research was supported by the Fundação de Amparo à Pesquisa do Estado de São Paulo under Grant FAPESP No. 92/4809-0. The authors are grateful to Dr. L. Cavaleri from Istituto Studio Dinamica Grandi Masse (Venice, Italy) for providing the data from the Gorbush Storm. The plots in this paper were made with the GrADS package developed by Brian E. Doty from the Center for Ocean-Land-Atmosphere Interaction. Helpful comments and suggestions received from two anonymous reviewers significantly improved the final version of this article. The Instituto de Estudos do Mar Almirante Paulo Moreira (Rio de Janeiro) gave us the motivation to develop the wave model and to study this case.

\section{REFERENCES}

Bannon, P. R., 1992: A model of Rocky Mountain lee cyclogenesis. J. Atmos. Sci., 49, 1510-1522.

Bates, J. R., and A. McDonald, 1982: Multiple-upstream, semi-Lagrangian advective schemes: Analysis and application to a mul- 
tilevel primitive equation model. Mon. Wea. Rev., 110, 1831 1842.

Breivik, L.-A., and M. Reistad, 1994: Assimilation of ERS-1 altimeter wave heights in an operational numerical wave model. Wea. Forecasting, 9, 440-451.

Businger, J. A., J. C. Wyngaard, Y. Izumi, and E. F. Bradley, 1971: Flux-profile relationships in the atmospheric surface layer. $J$. Atmos. Sci., 28, 181-189.

Davies, H. C., 1976: A lateral boundary formulation for multi-level prediction models. Quart. J. Roy. Meteor. Soc., 102, 405-418.

Dell'Osso, L., L. Bertotti, and L. Cavaleri, 1992: The Gorbush storm in the Mediterranean Sea: Atmospheric and wave simulation. Mon. Wea. Rev., 120, 77-90.

Gan, M. A., and V. B. Rao, 1991: Surface cyclogenesis over South America. Mon. Wea. Rev., 119, 1293-1302.

- and _ 1994: The influence of the Andes Cordillera on transient disturbances. Mon. Wea. Rev., 122, 1141-1157.

Geleyn, J. F., 1985: On a simple, parameter-free partition between moistening and precipitation in the Kuo scheme. Mon. Wea. Rev., 113, 405-407.

Golding, B., 1983: A wave prediction system for real-time sea state forecasting. Quart. J. Roy. Meteor. Soc., 109, 393-416.

Guillaume, A., 1990: Statistical tests for the comparison of surface gravity wave spectra with application to model validation. $J$. Atmos. Oceanic Technol., 7, 551-567.

Günther, H., W. Rosenthal, and M. Dunckel, 1981: The response of surface gravity waves to changing wind directions. J. Phys. Oceanogr., 10, 718-728.

Hasselmann, S., and K. Hasselmann, 1985: Computations and parameterizations of the nonlinear energy transfer in a gravity-wave spectrum. Part I: A new method for efficient computations of the exact nonlinear transfer integral. J. Phys. Oceanogr., 15, $1369-1377$

Innocentini, V., 1995: The Gorbush storm wave activity simulated by SAWMO model. Tech. Rep. 03-95, Instituto de Pesquisas Meteorológicas, Bauru, Brazil.

- and E. S. Caetano Neto, 1994: The SAWMO model: A second generation wave model applied to the South Atlantic. Tech. Rep. 01-94, Instituto de Pesquisas Meteorológicas, Bauru, Brazil.
James, I., and D. L. T. Anderson, 1984: The seasonal mean flow and distribution of large-scale weather systems in the Southern Hemisphere: The effects of moisture transports. Quart. J. Roy. Meteor. Soc., 110, 943-966.

Janssen, P. A. E., G. J. Komen, and W. J. P. de Voogt, 1984: An operational coupled hybrid wave prediction model. J. Geophys. Res., 89, 3635-3654.

Kuo, H. L., 1965: On formation and intensification of tropical cyclones through latent heat release by cumulus convection. $J$. Atmos. Sci., 22, 40-63.

Kuo, Y.-H., and R. J. Reed, 1988: Numerical simulation of an explosively deepening cyclone. Mon. Wea. Rev., 116, 2081-2105.

Mellor, G. L., and T. Yamada, 1974: A hierarchy of turbulence closure models for planetary boundary layers. J. Atmos. Sci., 31, $1791-1806$

Nagata, M., and Y. Ogura, 1991: A modeling case of interaction between heavy precipitation and a low-level jet over Japan in the Baiu season. Mon. Wea. Rev., 119, 1309-1336.

- M. Ikawa, S. Yoshizumi, and T. Yoshida, 1986: On the formation of a convergent cloud band over the Japan Sea in winter: Numerical experiments. J. Meteor. Soc. Japan, 64, 841-855.

Sanders, F., 1986: Explosive cyclogenesis in the west-central North Atlantic Ocean. Part I: Composite structure and mean behavior. Mon. Wea. Rev., 114, 1781-1794.

Sanders, J. W., W. J. de Voogt, and J. Bruinsma, 1981: Fysisch golfonderzoek noordzee. Sei. Rep. MLTP-2, Raad Overleg Fys. Oceanogr. Onderz. Noordzee, De Bilt, the Netherlands.

The SWAMP Group, 1985: Ocean Wave Modeling. Plenum Publishing, $256 \mathrm{pp}$.

Tatsumi, Y., 1983: An economical explicit time integration scheme for primitive model. J. Meteor. Soc. Japan, 61, 269-288.

van Vledder, G. P., and L. H. Holthuijsen, 1993: The directional response of ocean waves to turning winds. J. Phys. Oceanogr. 23, $177-192$.

Yamagishi, Y., 1980: Simulation of the air mass transformation process using a numerical model with the detailed boundary layer parametrization. J. Meteor. Soc. Japan, 58, 357-377.

Wash, C. H., R. A. Hale, P. H. Dobos, and E. J. Wright, 1992: Study of explosive and nonexplosive cyclogenesis during FGGE. Mon. Wea. Rev., 120, 40-51. 